\title{
Conditioned compensatory responses elicited by environmental signals for cyclophosphamide-induced suppression of antibody production in mice
}

\author{
MARVIN D. KRANK and GLENDA M. MACQUEEN \\ Mount Allison University, Sackville, New Brunswick, Canada
}

\begin{abstract}
Several investigators have reported that animals that receive taste cues paired with cyclophosphamide, an immunosuppressant drug, acquire a conditioned response that modifies the ability of the immune system to respond to antigenic stimulation (sheep red blood cells). Although most reports suggest that this conditioned response includes a suppression of antibody production, some investigators also have reported a conditioned enhancement of immune response. We investigated the conditioned response to environmental cues paired with cyclophosphamide. We discovered that, in contrast to the usual finding of conditioned suppression with taste cues, environmental cues elicit a compensatory conditioned response that reduces the residual suppression of antibody production caused by the drug. Additional evidence for compensatory conditioning was found in the relative weight gains of conditioned and unconditioned groups that were exposed to cyclophosphamide. Despite the clear development of a taste aversion in groups that were exposed to a taste cue in addition to the environmental cue, taste cues in compound with the environmental cues did not modify antibody production. These findings raise the possibility that previous reports of conditioned enhancement of immune response might be due to the presence of environmental cues.
\end{abstract}

In a series of recent experiments, Ader, Cohen, and their colleagues (for a review, see Ader \& Cohen, 1985) demonstrated that Pavlovian conditioning affects the ability of the immune system to respond to antigenic stimulation. In these studies, consumption of a saccharin solution is the conditioned stimulus (CS). This taste CS is followed by an injection of cyclophosphamide (Cy), an immunosuppressant drug, which serves as the unconditional stimulus (US). The acquisition of a conditioned response (CR) to the taste cue is measured by testing the ability of the taste CS to influence the response to external stimulation of the immune system. The typical finding in such studies is that the CR acquired to the taste cue is a suppression of the immune response to antigenic stimulation. For example, the immune system is less able to generate antibodies to an antigen when the animal is exposed to a taste cue that had been paired with $\mathrm{Cy}$.

Physiological CRs occurring in the immune system are important because they bear on basic theories about the nature of the CR in Pavlovian conditioning and the CR's potential regulatory role. As expected by Pavlov's (1927) theory of stimulus substitution, the conditioned suppression of antibody production by a taste cue that signals $\mathrm{Cy}$

This research was supported in part by a grant from the Natural Sciences and Engineering Research Council of Canada to the first author and by a Bell Research Fellowship from Mount Allison University to the second author. Requests for reprints should be addressed to Marvin D. Krank, Department of Psychology, Mount Allison University, Sackville, New Brunswick E0A 3C0, Canada. administration mimics the suppressant effect of the drug. That is, the CR affects the immune response in the same way that the drug affects the immune response. However, Pavlov's simple theory of the nature of the CR is complicated by demonstrations that environmental stimuli signaling drug administration often acquire the ability to elicit drug-opposite or compensatory CRs (for a recent review, see Siegel, Krank, \& Hinson, 1987). The demonstration of compensatory CRs in drug conditioning studies has led to the development of several modifications of Pavlov's original approach to the nature of the $\mathbf{C R}$.

The results of many drug conditioning studies with a variety of different response systems have been consistent with the predictions of Eikelboom and Stewart's (1982) homeostatic model and Hollis's (1982) adaptive response model of the CR. Eikelboom and Stewart (1982) argued that if a drug US disrupts peripheral homeostasis by affecting the efferent side of the nervous system, then the CR obtained should be a drug-opposite or compensatory response. Drug mimicking CRs are expected only when the drug exerts its effect centrally on the afferent side of the nervous system. From an ethological perspective, Hollis (1982) also argued that the drug CR normally serves the adaptive function of preparing the animal for the onset of the drug's adverse effects. According to this view, the CR functionally optimizes the interaction with the drug US.

At first glance, conditioned suppression of antibody production seems to contradict both these models. The 
conditioned suppression found by Ader and Cohen (1975, 1981) mimics, and would be expected to augment, $\mathrm{Cy}$ induced suppression. From their theoretical perspective, Eikelboom and Stewart (1982) would predict that the CR in these studies should be compensatory to this suppression; this is because $\mathrm{Cy}$, as an alkylating agent, directly prevents cell reproduction and probably suppresses immune response via cellular mechanisms independent of the central nervous system. Hollis's (1982) view of the $\mathrm{CR}$ as a biologically adaptive response also appears to be inconsistent with conditioned suppression. Because such suppression would normally be maladaptive by placing the animal at greater risk from foreign invaders, a CR that increases and augments such suppression would compound the maladaptive risk. If a CR served to "optimize" the interaction with the suppressant US, then this CR should be a compensatory adjustment to reduce this suppression.

Data consistent with homeostatic (Eikelboom \& Stewart, 1982) and adaptive (Hollis, 1982) models of the CR have been reported in studies of conditioning in the immune system (see Siegel et al., 1987). For example, Gorczynski, MacRae, and Kennedy (1984) reported that tastes paired with Cy may produce either conditioned suppression or conditioned enhancement of antibody production. They argue that the direction of the CR in this study was determined by the level of stress during conditioning, with greater levels of stress leading to the enhancement effect. Regardless of the explanation, the enhanced antibody production shown by some of Gorczynski et al.'s groups is consistent with a compensatory $\mathrm{CR}$ analysis. More importantly, these data indicate that the CR to signals for Cy may not be as straightforward as Ader and Cohen's (1985) recent review indicated (see comments by Hinson, 1985; Krank, 1985; Revusky, 1985).

One important difference between studies showing conditioned suppression of immune responses and studies demonstrating compensatory drug conditioning is the type of CS employed. Investigators of conditioned suppression of immune response have typically used taste cues, whereas investigators of compensatory drug effects have used environmental context cues (e.g., Siegel, Hinson, Krank, \& McCully, 1982). This difference may be important because many conditioning studies have stressed the importance of CS modality as a determinant of the nature of the CR (see cue-to-consequence specificity; Garcia \& Koelling, 1967). Moreover, because predictive environmental cues may often be present in studies investigating other nominal CSs, such as tastes, a CR to inadvertent environmental CSs may explain some of the variability in the direction of the CR reported by Gorczynski et al. (1984). This hypothesis is even more intriguing inasmuch as the potential signaling function of environmental cues is increased in studies presenting explicit stressors at the time of drug administrations.

The present study investigated the antibody production effects of a CR to an environmental CS paired with $\mathrm{Cy}$. The design of the study also assessed the effects of a taste
$\mathrm{CS}$ given in conjunction with the environmental cue. The taste CS $(0.1 \%$ saccharin) and the Cy dose $(100 \mathrm{mg} / \mathrm{kg})$ were chosen to replicate the parameters used in successful demonstrations of conditioned suppression and enhancement of antibody production in mice (e.g., Gorczynski et al., 1984). This procedure allows measurement of the interaction between the taste-activated CR and the environmental context-activated $C R$, and, thus, the results should be relevant to conflicting findings about the direction of the CR in studies of conditioned effects on antibody production.

\section{METHOD}

\section{Subjects and Apparatus}

Ninety-six male albino mice (CD[1]) from Charles River breeding farms, St. Constance, Quebec, were randomly assigned to 1 of 12 home cages. The animals were 50-60 days old at the start of the experiment. Each animal was given earpunch markings for group identification. The home cages were Plexiglas with cedar chip bedding. Food and water were available ad lib in the home cage except as described in the Procedure section below. The home colony room was illuminated from $0700-1900 \mathrm{~h}$ and dark from 1900-0700 h daily.

\section{Procedure}

This experiment consisted of two phases, conditioning and testing. During the conditioning phase, the mice received three conditioning trials. For 2 days prior to the start of a conditioning trial, the mice had water available for only $1 \mathrm{~h}$ per day. This was done to motivate saccharin consumption in mice exposed to the taste cue. Nevertheless, mice that were exposed to the environmental cue without the taste cue also were placed on this drinking schedule. Cue exposures for individual animals were at the same time each day $(0900,1130,1400$, or $1630 \mathrm{~h})$. On days prior to a cue exposure, water availability occurred at the same time as on the cue exposure day. Time-of-day variations were strictly counterbalanced between experimental conditions.

During each of the three conditioning trials, the mice were exposed to a designated cue for $1 \mathrm{~h}$. Half of the animals always received the environmental cue; the other half always received the environmental cue in conjunction with the saccharin taste cue. For the environmental cue used in the experiment, the animals were moved to a distinctive room and placed in a round, metal cage for a period of $1 \mathrm{~h}$. The distinctive room also was characterized by background music (maximum $80 \mathrm{~dB}$ ). The taste cue was a $0.1 \%$ $w / v$ solution of sodium saccharin. Taste cues, when presented, consisted of access to the saccharin solution in the distinctive environment for the entire 1-h period. These two CS types provided two replications of the conditioning procedures described below.

For each of the cue conditions, there were three experimental treatments during the conditioning phase (see Table 1). These differential treatments were defined by the relationship between the drug injection and the cue exposure. Two groups received injections of $100 \mathrm{mg} / \mathrm{kg}$ of $\mathrm{Cy}$. These $\mathrm{Cy}$ injections were either paired with cue exposure (i.e., given 15 min after the start of the cue exposure) or unpaired with cue exposure (i.e., given $4 \mathrm{~h}$ after the end of the cue exposure). A third group received saline injections paired with the cue exposure. At the completion of each trial, all animals were returned to their home cages and given free access to water. Conditioning trials were separated by 9 -day intervals to reduce the cumulative effects of drug administrations.

Fifteen days following the third conditioning trial, the test phase began. At the start of the test phase, each animal was inoculated with a .5-ml injection of a $1 \%$ thrice-washed solution of sheep red 
Table 1

Experimental Design Showing the Training and Testing Conditions for Each of the Four Groups

\begin{tabular}{lllcl} 
& & \multicolumn{3}{c}{ Testing Condition } \\
\cline { 4 - 5 } Group & Training Condition & Day 0 & Day 3 & Day 6 \\
\hline Paired & CS $\rightarrow$ Cy & SRBCs + CS & CS & Assay \\
Unpaired & CS $-4 \mathrm{~h} \rightarrow \mathrm{Cy}$ & SRBCs + CS & CS & Assay \\
Saline & CS - Sal & SRBCs + CS & CS & Assay \\
No CS & CS $\rightarrow$ Cy or & SRBCs & No CS & Assay \\
& CS $-4 \mathrm{~h} \rightarrow \mathrm{Cy} *$ & + No CS & & \\
\hline
\end{tabular}

Note $-n s=24$. CS refers to environmental cue alone for half of the animals and to environmental cue in conjunction with saccharin for the other half. $\mathrm{Cy}=$ cyclophosphamide. SRBC = sheep red blood cells. "Half of the animals in Group No CS received paired training; the other half received unpaired training.

blood cells (SRBCs) to stimulate antibody production. Immediately following inoculation, mice in Groups Paired, Unpaired, and Saline were reexposed to the same cue that had been used in training (environment CS or compound environment + taste CS) without any $\mathrm{Cy}$ or saline injection. Three days later each of these subjects was reexposed to the same conditioning cue (see Table 1). These groups of subjects differed only with respect to the cue-drug relationship during the training phase of the experiment; testing conditions were identical for these groups. The effects of conditioning should be revealed by the comparison of Group Paired and Group Unpaired. Both of these groups received equivalent exposure to both the $\mathrm{CS}$ and the drug during training. Because exposure during testing was also equivalent, differences in antibody production should reflect only differences due to the conditioning contingency.

A fourth group of animals (Group No CS) received only SRBC inoculation and were not exposed to the conditioning cue during the test phase. In Group No CS half of the animals had received the same training as Group Paired and half of the animals had received the same training as Group Unpaired. Because the CS was not presented during testing, this group served as an additional control for the residual effects of $\mathrm{Cy}$; that is, although the residual effects of Cy might suppress antibody production relative to Group Saline, Group No CS would not be expected to show the effects of conditioning during antibody production (see Ader \& Cohen, 1985; Gorczynski et al., 1984).

Six days after SRBC inoculation, each mouse was anesthetized with an injection of pentobarbital $(65 \mathrm{mg} / \mathrm{kg})$. Blood $(.5 \mathrm{cc})$ was sampled via cardiac puncture. The blood was centrifuged, and the obtained plasma was denatured by heating in a water bath at $56^{\circ} \mathrm{C}$ for $30 \mathrm{~min}$. The plasma was then analyzed for antibodies specific to SRBC by means of passive hemaglutination. The resulting antibody titer represented a log base 2 measure of antibody concentration and served as the dependent measure of immune system response. In addition to this measure of antibody production, we also monitored weight changes in each group and saccharin consumption in groups that had received the taste cue exposures.

Other than the use of an explicit environmental context manipulation, the procedures used in this study for the most part replicate those used by Gorczynski et al. (1984), with the following exceptions: We used a different strain of mice, our deprivation schedule was intermittent rather than continuous, the mice received only two instead of three CS reexposures following antigenic stimulation. the exposure on day 3 that we used is normally used by Ader and Cohen (see review, 1985), and our assay was the less sensitive antibody titer method used by Ader and Cohen (1975) rather than the plaque forming cell method used by Gorczynski et al. None of these differences would be expected to modify the influence of conditioning on antibody production in any qualitative manner.

\section{RESULTS}

\section{Immune Response}

Table 2 shows the average $\log _{2}$ titer for passive hemaglutination in each of the four groups for both CS types. A two-way analysis of variance (ANOVA) with group and CS type as factors revealed a significant main effect of group $[F(3,88)=5.16, p<.01]$, but no main effect of saccharin presentation $[F(3,88)<1]$ or interaction with saccharin presentation $[F(3,88)<1]$. Newman-Keuls pairwise tests $(p<.05)$ revealed the following ordering among the groups: Paired $=\mathrm{Sa}$ line > No CS > Unpaired. These analyses demonstrated that Group Paired animals, which had received an environmental signal for $\mathrm{Cy}$ and were reexposed to that signal following SRBC inoculation, produced higher antibody levels than did either Group Unpaired animals, which had received experience with the environmental cue explicitly unpaired with $\mathrm{Cy}$, or Group No CS animals, which had received the same environmental signal for $\mathrm{Cy}$ during training but were not reexposed to that signal following SRBC inoculation. Group Paired antibody titers did not reveal any residual suppression by previous experience with $\mathrm{Cy}$ because they were not different from Group Saline antibody titers. Both Group No CS and Group Unpaired evidenced $\mathrm{Cy}$-induced suppression of antibody production compared with Group Saline. Group No CS antibody production was, however, less sup-

Table 2

Mean $( \pm S E M)$ Antibody Titer $\left(\log _{2}\right)$ as a Function of Training Condition and Conditioned Stimulus (CS) Type

\begin{tabular}{lcccc}
\hline \multirow{2}{*}{ CS Type } & \multicolumn{4}{c}{ Group } \\
\cline { 2 - 5 } \multicolumn{1}{c}{ Paired } & Unpaired & Saline & No CS \\
\hline Environment & $4.91 \pm .34$ & $3.50 \pm .26$ & $4.36 \pm .41$ & $4.08 \pm .29$ \\
Environment + Saccharin & $4.50 \pm .19$ & $3.63 \pm .34$ & $4.67 \pm .33$ & $4.00 \pm .33$ \\
\hline
\end{tabular}


pressed than Group Unpaired production. Further breakdown of Group No CS according to training condition indicates that the effects of conditioning are specific to the CS presentation during the test phase. When scores for Group No CS were collapsed across CS type, unpaired trained animals $(n=12, M=3.92)$ did not differ from paired trained animals $(n=12, M=4.17)$.

\section{Weight Changes}

Another indication of an effect of conditioning with $\mathrm{Cy}$ was shown by the record of weight change during the conditioning phase. By the end of the conditioning phase, mice that received paired $\mathrm{Cy}$ administrations (Group Paired, $n=24$, and half of Group No CS, $n=12$ ) gained a mean of $5.9 \mathrm{~g}$, and mice that received unpaired Cy administrations (Group Unpaired, $n=24$, and half of Group No CS, $n=12$ ) gained a mean of $4.9 \mathrm{~g}$. Mice that received only saline injections (Group Saline, $n=24$ ) gained a mean of $7.8 \mathrm{~g}$. Because the animals' weights did not differ at the beginning of conditioning [the means at the start of conditioning were $35.7 \mathrm{~g}, 33.8 \mathrm{~g}$, and $34.4 \mathrm{~g}$, respectively; $F(2,90)<1$ ], an unequal $n$ ANOVA for the weight change effects of conditioning with $\mathrm{Cy}$ was conducted, using the difference between each animal's weight on the first conditioning trial (prior to Cy administration) and its weight on the first test trial (prior to SRBC inoculation). The ANOVA revealed a significant effect of training condition on weight change $[F(2,90)=8.03$, $p<.001]$. According to the Newman-Keuls pairwise test, saline animals that had not been injected with $\mathrm{Cy}$ gained more weight than either of the two groups of animals that had received Cy. In addition, Group Paired animals gained more weight than Group Unpaired animals. The presence of saccharin had no main effect $[F(2,90)<1]$ and did not interact with the training condition effect $[F(2,90)=1.08]$.

Overall, individual weight changes and antibody titers were not correlated (Pearson product moment correlation, $r=.03)$. However, when calculated within groups, weight changes in animals that received unpaired training were positively correlated with antibody titers $[r=$ $.43, p<.01]$. Moreover, when further subdivided according to CS type, this correlation was evident only in those animals trained with the environment cue alone $[r=$ $.83, p<.001]$. The reason for this correlation and its relevance to conditioned effects on antibody production are unclear, as the presence of the saccharin cue had no significant effects on either weight changes or antibody titers.

\section{Taste Aversion}

Saccharin consumption during the two CS reexposures was measured for those animals in Group Paired, Group Unpaired, and Group Saline that were trained and tested with the saccharin available (see Table 3 ). Consumption (in milliliters) of the saccharin solution was analyzed by a two-way mixed-design ANOVA, with training condition as a between factor and CS reexposure (first vs. sec-
Table 3

Mean Saccharin Consumption (in ml) on the Two Test Presentations Following Sheep Red Blood Cell Inoculation

\begin{tabular}{cccc}
\hline & \multicolumn{3}{c}{ Training Condition } \\
\cline { 2 - 4 } Saccharin Test & Paired & Unpaired & Saline \\
\hline 1 & 2.37 & 4.03 & 4.99 \\
2 & 2.49 & 2.40 & 4.41 \\
\hline
\end{tabular}

ond) as a within factor. The ANOVA revealed a significant effect of training condition $[F(2,33)=9.30$, $p<.001]$, a significant effect of CS test $[F(1,33)=6.84$, $p<.025]$, and a CS test $\times$ training condition interaction $[F(2,33)=3.67, p<.001]$. To further delineate the nature of the interaction effect, separate analyses of intake levels on each CS reexposure were performed. Consumption levels differed on both the first and the second CS reexposures $[F(2,33)=8.51$ and 9.73 , respectively, both $p s<.001]$. On the first reexposure test, Group Paired drank significantly less saccharin than Group Saline and Group Unpaired (Newman-Keuls test, $p<.05)$. Although Group Unpaired seemed to have lower consumption levels than Group Saline, these two groups did not differ. On the second CS reexposure, Group Paired and Group Unpaired did not differ, but both groups drank less saccharin than Group Saline (Newman-Keuls test, $p<.05$ ). Neither the first nor the second reexposure consumption values correlated with antibody titers.

\section{DISCUSSION}

The present results demonstrate three effects of conditioning with $\mathrm{Cy}$ as a US. Of primary interest is the observation that an environmental signal paired with $\mathrm{Cy}$ controls a compensatory reduction in Cy's residual suppression of the immune system's ability to respond to antigenic stimulation. This result contrasts with the usual finding of conditioned suppression of antibody production to taste cues paired with Cy (Ader \& Cohen, 1975, 1981). The results also suggest that conditioning processes aid in the resistance to $\mathrm{Cy}$-induced reductions in weight gain. Finally, the presence of a saccharin taste CS in conjunction with the environmental CS results in the acquisition of a conditioned taste aversion, but does not affect the expression of environmental CS mediated effects.

Evidence for a conditioned compensation for $\mathrm{Cy}$ induced suppression of immune function is found in the superior antibody production by mice that were reexposed to the environmental cue for Cy following inoculation with SRBCs. The Group Paired mice produced higher levels of antibodies than mice that either were not reexposed to the environmental cue (Group No CS) or had explicitly unpaired training with the environmental cue and $\mathrm{Cy}$ (Group Unpaired). Despite the fact that Group Paired mice had been given doses of $\mathrm{Cy}$ that produced residual suppression of antibody production in other groups, Group Paired antibody levels were indistinguishable from those of control mice (Group Saline) whose immune systems 
were unchallenged by $\mathrm{Cy}$-induced immunosuppression. These comparisons indicate that the CS presentation following SRBC inoculation compensates for the residual suppressant effects of $\mathrm{Cy}$.

Additional evidence for the compensatory effects of the environmental signal for $\mathrm{Cy}$ is found in the weight changes among the individual groups. Animals administered Cy gained less weight than saline control animals. Paired animals, however, were more resistant to this adverse drug effect. Krank, Hinson, and Siegel (1984) reported a similar effect of conditioning on the weight gain effects of morphine. In their study, rats that received a contingent signal for each morphine administration gained more weight than rats that received the same number and dose of morphine administrations but had the contingency between the signal and the drug reduced by partial reinforcement procedures. The present observation that weight gain in mice is less affected by explicitly signaled injections of $\mathrm{Cy}$ than by unsignaled injections can be interpreted as the cumulative effect of compensatory responses that allow the animal to better cope with the drug's adverse impact.

The relationship between the weight change score and antibody titers is unclear. Although evidence for a sizable positive correlation between these measures is found in one subgroup (unpaired training with the environmental CS), all other groups showed slightly negative and nonsignificant correlations. Given that the presence of the saccharin affected neither weight changes nor antibody titers, this observation is enigmatic. The present study is unique in assessing the weight changes as a function of conditioning experience with $\mathrm{Cy}$. Obviously, further research specifically aimed at assessing the relationship between these two measures will be needed to clarify this issue.

Regardless of the relationship between the immune system response and weight gain, the environmental signal for $\mathrm{Cy}$ reduces the disruptive effects of the drug on both of these measures. Although no direct measures of compensatory CRs were taken, these observations are consistent with predictions derived from Eikelboom and Stewart's (1982) homeostatic model and Hollis's (1982) adaptive response model of the CR. Both the immune system response and weight gain effects of the signal serve to reduce the disruptive effects of $\mathrm{Cy}$ and, thus, tend to restore the body's homeostatic balance and optimize the interaction with the drug. Moreover, because the reduction of Cy's adverse effects functionally represents tolerance, the present findings are consistent with a large body of evidence showing that conditioning processes contribute to drug tolerance.

Additional evidence of conditioning is found in the saccharin consumption of those animals that had been exposed to the taste cue. On the first CS test, paired animals drank less saccharin than either unpaired or saline control animals. This observation confirms the ability of $\mathrm{Cy}$ administration to support a conditioned taste aversion. On the second CS test, the paired group drank less saccharin than the saline control group. However, the unpaired group also drank less saccharin than the saline control group and, in fact, did not differ from the paired group on this test. One possible explanation for this finding is that the SRBC administration is itself capable of supporting a weak taste aversion. Some evidence in support of this possibility is that both unpaired and control animals drank less saccharin on the second test. Paired animals may not have shown this effect because they already had suppressed levels of consumption. Unpaired animals may have been especially susceptible to the weak aversionproducing agent because of sensitization or a mild aversion from previous experience with $\mathrm{Cy}$. A mild taste aversion in the unpaired animals would be expected to the extent that the 4-h delay before the $\mathrm{Cy}$ administration was insufficient to eliminate long delay learning (Revusky \& Garcia, 1970) and may be revealed by the nonsignificant trend toward lower consumption than the control group on the first test.

Despite the development of a conditioned taste aversion, no evidence was found for conditioned suppression of antibody production by the taste cue. This finding contrasts with the observations of Ader and Cohen (1975, 1981), but confirms the observations of Gorczynski et al. (1984) that the CR to a signal for Cy is not always suppression of immune function. As in the Gorczynski et al. experiments, the conditioned animals show enhancement only when compared with animals whose immune systems are compromised by previous $\mathrm{Cy}$ administrations and not when compared with saline control animals. Nevertheless, these data are consistent with the notion that the environmental CS controls a compensatory reduction in the residual effects of $\mathrm{Cy}$, which may interact with any conditioning to the taste CS.

Although explicit control of pairings of $\mathrm{Cy}$ with an environmental CS was the main difference between this study and previous demonstrations of conditioned suppression to taste CSs, several other differences should be considered. The design of the present study did not quite replicate that of either Gorczynski et al. (1984) or Ader and Cohen (1975). Details of these differences, most of which were minor, are listed in the Method section. One that may be important, however, was our deprivation schedule. Unlike previous studies, our animals were not maintained on a constant water-deprivation schedule. Instead, we allowed our animals free access to water between $\mathrm{Cy}$ injections until $48 \mathrm{~h}$ prior to the next injection. Because deprivation may affect the animal's physiological and psychological status in many ways, this difference may be important.

A second explanation of our failure to find conditioned suppression of antibody production to a taste CS is that stress may interact with conditioning of immune responses (Gorczynski et al., 1984). As with most studies of environmental conditioning, the contextual cue used in this study probably evoked a stress reaction. Indeed, such a stress reaction might explain why antibody titers in Group No CS were superior to antibody titers in Group Unpaired. Sato, Flood, and Makinodan (1984) showed that 
mild stress reduces antibody production only in animals compromised by X-ray irradiation. Because Group Unpaired was both compromised by its exposure to $\mathrm{Cy}$ and stressed by its exposure to the environmental context, this group would be expected to have lower antibody titers than Group No CS which, although compromised by its exposure to $\mathrm{Cy}$, was not reexposed to the environmental context.

Although differential stress may help to explain the difference between Group No CS and Group Unpaired, it is unclear how this would account for the effects of conditioning found in Group Paired. From the stress interaction perspective, the conditioning effect on antibody titers found in Group Paired is even more impressive. Like Group Unpaired, Group Paired was both compromised by its exposure to $\mathrm{Cy}$ and stressed by its exposure to the environmental context, yet the antibody titers revealed in Group Paired do not differ from antibody titers in uncompromised animals from Group Saline or in compromised animals from Group No CS that were not reexposed to the environmental context.

A different type of stress effect was proposed by Gorczynski et al. (1984), who argued that stress modifies the natural physiological state of the animal at the time of experimentation. The direction of conditioning, then, reflects the basic physiological state of the organism. Although the mechanism for producing a complete reversal in the direction of the $C R$ is unspecified, the present findings could be due to such a modification of the internal milieu. Clearly, the exposure to a stressful environmental context could provide the basis for modifying any of a variety of neuroendocrine systems that are known to interact with the immune system (e.g., Besedorsky \& Sorkin, 1981). It is important to note, however, that in the present study both Group Paired and Group Unpaired were equally exposed to the environmental context and the immunosuppressant drug. Any explanation based on stressinduced modifications must account for the fact that a delay interval of only $4 \mathrm{~h}$ eliminates the effect.

We prefer an alternative explanation of the failure to find conditioned suppression to the taste CS based on wellestablished principles of conditioning (Krank, 1985; Siegel et al., 1987). This explanation relies on the welldocumented observation of cue-to-consequence specificity (Garcia \& Koelling, 1967) and its potential influence on the nature of the $C R$ in the immune system. Drug conditioning studies that have reported compensatory CRs (Siegel, 1983; Siegel et al., 1987) have used environmental CSs rather than taste CSs. Studies with taste CSs as signals for drugs have typically assessed the development of conditioned aversions (e.g., Cappell, LeBlanc, \& Endrenyi, 1973). A recent study demonstrated that tastes associated with ethanol do not affect ethanol tolerance, even though evidence for the association is revealed by a taste aversion (Cunningham \& Hallett, 1988). These observations suggest the possibility that conditioned suppression with Cy as the US may be unique to the use of a taste CS. Environmental signals for Cy may result in the ac- quisition of a different, possibly compensatory, CR. Although Dyck, Greenberg, and Osachuk (1986) used a different immune response, their data provide some additional support for the suggestion that environmental cues can control compensatory conditioning in the immune system. Using an environmental CS, they observed a CR that compensated for Poly I:C-induced activation of natural killer cell activity.

Cue-to-sequence specificity may explain why the CR to environmental signals is compensatory, but it does not account for the failure to observe suppression of antibody production to the taste cues when they are present. Conditioned immunosuppression may not have been found with the taste cue used in this experiment because of overshadowing (Kamin, 1969). Because the taste cue was presented only in compound with the environmental cue, the taste cue does not uniquely predict $\mathrm{Cy}$. Under such conditions, the more salient stimulus will acquire a much stronger association than will the less salient stimulus. To the extent that our environmental manipulation, which involved multiple modalities, was more salient than the taste cue for immune conditioning, the environmental cue would overshadow the taste cue. Moreover, even if the taste cue acquired the ability to suppress antibody production, the presence of an environmental cue and its compensatory $\mathrm{CR}$ at testing could mask this response.

In general, this analysis suggests that closer attention should be paid to the presence of environmental cues in studies of conditioning in the immune system. For example, overshadowing by more salient environmental cues may have been a factor in the Gorczynski et al. (1984) studies. They found that the direction of the CR to a taste CS was a function of time of day and the presence of external stress. Inadvertent environmental cues may confound any conditioning study, but studies that specifically vary time of day and physical stress will necessarily introduce concomitant environmental cues that may interact with other conditioning contingencies. Such environmental cues will compete with the taste CS for association with the Cy US. Moreover, to the extent that the CRs to taste CSs and environmental CSs are opposite each other, the specific contingency could result in either conditioned suppression of immune function when the taste CS gains the most associative strength or conditioned compensation of immune function when the environmental CS gains the most associative strength.

\section{REFERENCES}

Ader, R., \& COHEN, N. (1975). Behaviorally conditioned immunosuppression. Psychosomatic Medicine, 37, 333-340.

ADER, R., COHEN, N. (1981). Conditioned immunopharmacologic responses. In R. Ader (Ed.), Psychoneuroimmunology (pp. 281-319). New York: Academic Press.

ADER, R., COHEN, N. (1985). CNS-immune system interactions: Conditioning phenomena. Behavioral \& Brain Sciences, 8, 379-394.

BESEDORSKY, H. O., SorkIN, E. (1981). Immunologic-neuroendocrine circuits: Physiologic approaches. In R. Ader (Ed.), Psychoneuroimmunology (pp. 545-574). New York: Academic Press. Cappell, H., LeBlanc, A. E., Endrenyi, L. (1973). Aversive con- 
ditioning with psychoactive drugs: Effects of morphine, alcohol and chlordiazepoxide. Psychopharmacology, 3, 239-246.

Cunningham, C. L., \& Hallett, C. L. (1988). Flavor cues mediate ethanol-induced taste avoidance but not conditioned tolerance. Alcoholism: Clinical \& Experimental Research, 12, 310.

Dyck, D. G., Greenberg, A. H., \& Osachuk, T. A. G. (1986). Tolerance to drug-induced (Poly $\mathrm{I}: \mathrm{C}$ ) natural killer (NK) cell activation: Congruence with a Pavlovian conditioning model. Journal of Experimental Psychology: Animal Behavior Processes, 12, $25-31$.

EIKElBoOM, R., \& SteWART, J. (1982). Conditioning of drug-induced physiological responses. Psychological Review, 89, 507-528.

GarCIA, J., Koelling, R. A. (1967). The relationship of cue consequence in avoidance learning. Psychonomic Science, 4, 123-124.

GorCZYNSKI, R. M., MACRAe, S., \& KenNedy, M. (1984). Factors involved in the classical conditioning of antibody responses in mice. In R. Ballieux, J. Fielding, \& A. L'Abbatte (Eds.), Breakdown in human adaptation to stress: Towards a multidisciplinary approach (pp. 704-712). Boston: Kluwer-Nijhoff.

Hinson, R. E. (1985). Conditioned immunosuppression and the adaptive function of Pavlovian conditioning. Behavioral \& Brain Sciences, $8,403$.

HoLLus, K. (1982). Pavlovian conditioning of signal-centered action patterns and autonomic behavior: A biological analysis of function. In J. S. Rosenblatt, R. A. Hinde, \& C. Beer (Eds.), Advances in the study of behavior (Vol. 12, pp. 1-51). London: Academic Press.

Kamin, L. J. (1969). Predictability, surprise, attention and conditioning. In B. A. Campbell \& R. M. Church (Eds.), Punishment and aversive behavior (pp. 279-296). New York: Appleton-Century-Crofts.

KRANK, M. D. (1985). Conditioning the immune system: New evidence for the modification of physiological responses by drug-associated cues. Behavioral \& Brain Sciences, 8, 405-406.

Krank, M. D., Hinson, R. E., \& Siegel, S. (1984). The effect of partial reinforcement on tolerance to morphine-induced analgesia and weight loss in the rat. Behavioral Neuroscience, 98, 79-85.

Paviov, I. P. (1927). Conditioned reflexes (G. V. Anrep, Trans.). London: Oxford University Press.

REVUSKY, S. (1985). Questions about conditioned immunosuppression and biological adaptation. Behavioral \& Brain Sciences, 8, 407.

REVUSKY, S. H., GARCIA, J. (1970). Learned association over long delays. In G. H. Bower (Ed.), The psychology of learning and motivation (Vol. 4, pp. 1-84). New York: Academic Press.

SAto, K., Flood, J. F., Makinodan, T. (1984). The influence of conditioned psychological stress on immunological recovery in mice exposed to low dose X-irradiation. Radiation Research, 98, 381-388.

Siegel, S. (1983). Classical conditioning, drug tolerance, and drug dependence. In Y. Israel, F. B. Glaser, H. Kalant, R. E. Popham W. Schmidt, \& R. G. Smart (Eds.), Research advances in alcohol and drug problems (Vol. 7, pp. 207-246). New York: Plenum Press.

Siegel, S., Hinson, R. E., Krank, M. D., McCully, J. (1982). Heroin "overdose" death: The contribution of drug-associated environmental cues. Science, 216, 436-437.

Siegel, S. KRANK, M. D., \& Hinson, R. E. (1987). Anticipation of pharmacological and non-pharmacological events: Classical conditioning and addictive behavior. Journal of Drug Issues, 1, 83-110.

(Manuscript received December 8, 1987; revision accepted for publication July 11,1988 .) 